\title{
Educación en diabetes: un aspecto clave de la formación actual en enfermería
}

\author{
Education regarding diabetes: a key aspect in current nursing training
}

Educacao em diabetes: um fator chave da formação em enfermagem

\section{Ruth F. Hernández-Páez ; Luz H. Aponte-Garzón ${ }^{1}$}

Enfermera, MSc. Grupo de investigación de Cuidado. Docente Facultad de Ciencias de la Salud, Programa de Enfermería, Universidad de los Llanos.

Email: grupocuidado@unillanos.edu.co

\section{Resumen}

Considerando la enorme importancia de la diabetes Mellitus como enfermedad metabólica crónica, a nivel global como local, se plantea la presente revisión en el marco del proyecto titulado Conocimiento de los profesionales de enfermería sobre diabetes Mellitus tipo 2 en las áreas clínica y comunitaria de Villavicencio, Colombia. El objetivo, describir los requerimientos de educación continuada de profesionales de enfermería para asistir a personas con Diabetes Mellitus 2, en aspectos relacionados con promoción de la salud. El estudio marco de la revisión, muestra una aproximación a la realidad de la prestación del servicio de enfermería, señalando fragilidad en los conocimientos de los profesionales de enfermería, en los aspectos básicos que requiere la atención de las personas con Diabetes Mellitus 2, durante el transcurso de su vida una vez diagnosticadas, subrayando la necesidad de actualizar a los profesionales para mejorar el cuidado en el ejercicio de la profesión y mejorar las formas de la educación en salud. Se determinó presentar una propuesta educativa cuyas temáticas se fundamentan a través de la revisión. Se plantean fundamentos que podrían incluirse en un curso de actualización en Diabetes para profesionales de enfermería, orientado a fortalecer conocimientos sobre la enfermedad y el cuidado de las personas con Diabetes Mellitus 2. Se enfatiza la promoción de la salud y la visión educativa centrada en la persona con la enfermedad, entendiendo la importancia de su conocimiento en el logro del control metabólico, la prevención de complicaciones y el mejoramiento de la calidad de vida.

Palabras Clave: diabetes mellitus, educación en enfermería, promoción de la salud (fuente: $\mathrm{MeSH})$. 


\begin{abstract}
Considering the enormous importance of diabetes mellitus as achronic metabolic disease, at both global and local level, the present review was set within the framework of a project titled, "Qualified nurses' knowledge regarding diabetes mellitus type 2 in clinical and communitybased areas of Villavicencio, Colombia." The objective was to describe qualified nurses' requirements regarding their further education related to attending people suffering diabetes mellitus type 2, concerning aspects related to promoting healthy life styles. The study involved an approach to the reality of providing nursing services; it pointed out weakness in qualified nurses' knowledge regarding basic aspects required for attending people suffering from diabetes mellitus 2 during their lives after having been diagnosed, high lighting the need for updating qualified nurses to improve care when practising their profession and improving the types of health education. It was determined that an educational proposal should be presented whose themes were grounded on the review. Fundamental points were proposed which could be included in a course for qualified nurses aimed at up-dating their knowledge regarding diabetes, orientated towards strengthening knowledge about the disease and caring for people suffering from diabetes mellitus 2. This would emphasise promoting healthy lifestyles and an educational view point centred on people suffering the disease, understanding the importance of such knowledge in achieving metabolic control, preventing complications and improving the patients' quality of life (as well as that of the caregivers).
\end{abstract}

Key words: diabetes mellitus, nursing education, promoting a healthy lifestyle (source: MeSH).

\title{
Resumo
}

Em consideração á grande papel da diabetes mellitus como doença metabólica crônica, se apresenta esta revisão como parte do projeto "conhecimento dos profissionais de enfermagem sobre a diabetes mellitus tipo 2 nas áreas clinica e comunitária de Villavicencio, Colombia". O objetivo descrever os requerimentos de educação continua de profissionais de enfermagem para atender a pessoas com Diabetes Mellitus 2, em tópicos relacionados com a promoção da saúde. O estudo apresenta, uma aproximação á realidade da prestação do serviço de enfermagem, indicando fragilidade nos conhecimentos dos professionais de enfermagem, nos assuntos básicos que requer a atenção das pessoas com Diabetes Mellitus 2, ao longo da sua vida após serem diagnosticados, salientando a necessidade de atualizar aos profissionais para melhorar o cuidado no exercício da profissão e melhorar uma proposta educativa com temáticas fundamentadas nesta revisão. Propõem-se tópicos que devem ser incluídos num cursinho de atualização em diabetes para profissionais em enfermagem visando fortalecer os conhecimentos na doença e o cuidado das pessoas com diabetes mellitus 2. É enfatizado a promoção da saúde, e a visão educativa tendo como eixo a pessoa com a doença, considerado o valor do ser conhecimento no logro do controle metabólico, a prevenção de complicações e o melhoramento da qualidade de vida.

Palabras Clave: Diabete mellitus, educação em enfermagem, promoção da saude (fuente: $\mathrm{MeSH})$. 


\section{Introducción}

La diabetes es una enfermedad metabólica crónica epidémica catalogada como el tercer problema mundial de salud pública por su incidencia; 347 millones de personas en el mundo están comprometidas con la enfermedad y más del $80 \%$ de ellas pertenecen a grupos de ingresos medios y bajos. La hiperglucemia es el efecto común de la diabetes no controlada, que con el tiempo ocasiona graves lesiones en el sistema nervioso y cardiovascular principalmente, con riesgo adicional de complicaciones renales, neuropáticas, de visión y de amputación de miembros inferiores.

La Organización Mundial de la Salud (OMS, 2011) ha proyectado que las muertes por diabetes se duplicarán entre el 2005 y el 2030. Por lo cual, dicha organización tiene como misión prevenir la diabetes siempre que sea posible, y cuando no sea posible, minimizar las complicaciones y mejorar la calidad de vida de las personas con la enfermedad. Se requiere, entonces, desde los sistemas de salud, elevar la conciencia del problema, fomentar la prevención y el control de la enfermedad.

En Colombia, la tasa de mortalidad por Diabetes Mellitus para el año 2008 fue de 66.7/100.000 habitantes mayores de 45 años, tasa que ha venido en aumento, ocupando el cuarto lugar dentro de las causas de mortalidad por enfermedades crónicas, en el mismo año (2008). El Plan Nacional de Salud Pública 2007 - 2010, plantea el enfoque de atención desde los determinantes de la salud, con la intención de intervenir los factores que por su naturaleza son modificables, con el concurso de los actores de salud, de otros sectores comprometidos y con la comunidad.

En consecuencia, la promoción de la salud busca facilitar los medios para que las personas transformen los comportamientos individuales y grupales, las condiciones del entorno y de los servicios que afectan la salud y la calidad de vida, haciendo posible la vinculación de los temas de salud con los temas de otros sectores relacionados con la política económica y social. En 1978 la OMS, con el patrocinio del Fondo de las Naciones Unidas para la Infancia (UNICEF), proclamó en Alma-Ata la salud como derecho humano fundamental y el logro del grado más alto posible de salud, un objetivo muy importante. Los gobiernos debían procurar para el año 2000 brindar salud para todos los pueblos del mundo (Higashida, 2008).

El milenio terminó y no fue alcanzada la meta propuesta en Alma-Ata. Las enfermedades crónicas como la diabetes han impactado enormemente la salud de las personas y sus familias, dado los factores asociados con los estilos de vida cambiantes en el orden mundial y los relacionados también con el envejecimiento de la población.

Por otra parte, en el análisis del contexto regional, la formación de los profesionales de enfermería, quienes deben atender en gran medida este problema, se orienta sobre estrategias de atención a los problemas de salud de alta relevancia en el país, a través de guías y normas de atención específica; muchas veces requirieren de actualización para la asistencia profesional, en programas especiales, como la atención a las personas con enfermedad crónica; entre ellas, las personas con Diabetes Mellitus 2, DM2 (UNILLANOS, 2010). Esta situación hace necesario enfatizar en los conocimientos fisiopatológicos básicos para atender las enfermedades crónicas de alta exigencia, como en los conocimientos que fundamentan la importancia de la relación entre los profesionales de enfermería y sus pacientes. Se debe entender que su participación activa es indispensable para el desarrollo del autocuidado, para el mantenimiento de su salud y de su calidad de vida. 
Dada la relevancia del tema en Villavicencio, Meta, durante el año 2011 se efectúo un estudio para la evaluación de conocimientos de los profesionales sobre la DM2, por docentes del Programa de Enfermería de la Universidad de los Llanos (Aponte-Garzón y Hernández-Páez, 2012). Se hallaron bajos conocimientos para la atención de las personas con la enfermedad. En forma complementaria, se señalaron las actuales exigencias de las instituciones de salud, en las que los profesionales de enfermería se ven enfrentados a priorizar el registro de acciones. Ellos se ocupan más de los sistemas de información institucionales y de la gestión administrativa, que del ofrecimiento del cuidado directo de la salud a las personas en forma individualizada y colectiva.

El análisis de los resultados del estudio mencionado en Villavicencio sobre conocimientos de los profesionales de enfermería en DM2 que asisten a personas con la enfermedad en las áreas clínica y comunitaria, da cuenta de la fragilidad de los conocimientos en los diferentes aspectos básicos que requieren la atención de una persona con la enfermedad, durante el transcurso de su vida, después de haber sido diagnosticada. Un porcentaje bajo de los profesionales ha logrado conocimientos en algunos de los aspectos relacionados con el diagnóstico y la fisiopatología, los factores de riesgo, las complicaciones agudas y crónicas, el tratamiento farmacológico y no farmacológico y las medidas preventivas para no desarrollar la enfermedad y evitar desarrollar complicaciones. Es evidente la necesidad de actualizar a los profesionales de enfermería en los aspectos enunciados para mejorar el cuidado en el ejercicio diario de la profesión y, mejorar las formas en las que se logra la educación en salud; de manera que ésta pueda ser oportuna, idónea, responsable, completa y ante todo sensible a las necesidades de cada una de las personas que vivencian la condición de cronicidad (Miyar et al., 2008).
Así, a partir de los resultados del estudio denominado Conocimiento de los profesionales de enfermería sobre diabetes Mellitus tipo 2 en las áreas clínica y comunitaria, Villavicencio, Colombia, se incluye una propuesta educativa como estrategia de formación continuada, dirigida a profesionales de enfermería, para fortalecer los conocimientos sobre la enfermedad y las respuestas a las necesidades de cuidado, con una visión centrada en el paciente. Finalmente, se busca lograr mejor adherencia al tratamiento y mejor calidad de vida de las personas con diabetes, con la debida asistencia de enfermería. Para lograr lo anterior, se debe entender que los cambios de comportamiento esperados en ellas, dependen en gran medida de la comprensión, destreza y motivación que tengan para su autocuidado; y al mismo tiempo, de las capacidades de los profesionales de la salud para lograrlo.

A continuación se dan algunos fundamentos que podrían incluirse en un curso de actualización en Diabetes para profesionales de enfermería en la Orinoquía Colombiana.

\section{Educación terapéutica}

La salud es un fenómeno complejo y dinámico que se constituye como producto de sus determinantes sociales, biológicos y psicológicos, concepto descrito por Lalonde (1974). Investigadores preocupados por el desarrollo de la salud pública, diseñaron un instrumento para mapear las condiciones que determinan los ambientes de salud. Allí se muestra la importancia de la construcción de ambientes saludables, facilitada con el empoderamiento de los actores de salud, en la producción de acciones desde otros sectores, tales como el social y el económico a su favor. Esta concepción contribuye, en tanto visión, al ejercicio profesional de la salud, como estrategia para que las acciones en salud trasciendan e involucren el compromiso de otros sectores; en el apoyo para la generación 
de cambios de comportamiento o mejoramiento de estilos de vida, renovando la percepción de la salud, al ser concebida como un todo complejo (Commers et al., 2006).

Desde esta mirada previa de la salud, se hace también necesario visualizar un modelo de intervención que logre atender los problemas relacionados específicamente con la cronicidad, característica de la diabetes; que desde una perspectiva de desarrollo integral, valore la dimensión educativa a largo plazo para el mantenimiento de la salud y el bienestar. En este sentido, la educación terapéutica ha desarrollado concepciones para mejorar la educación en salud, proponiendo un cambio en las relaciones entre el profesional de la salud y la persona con la enfermedad, en donde el primero no dirige, ni interviene y ni controla directamente el tratamiento, sino que establece un contacto terapéutico con la persona con la enfermedad, sobre la que recae una importante responsabilidad de su cuidado.

En esta perspectiva, es necesario fortalecer el conocimiento de los profesionales de la salud, no solo en los aspectos de atención médicosanitaria, sino también en otras habilidades desarrolladas por las disciplinas sociales y de la conducta, para lograr desde la asistencia en salud, personas más responsables y capaces ante la enfermedad (Mendoza et al., 2010).

La educación terapéutica ha sido conceptuada por la OMS (1998), como la educación para formar, convencer, motivar y fortalecer a las personas con enfermedades no transmisibles (ENT), para que participen activamente en su tratamiento y puedan afrontar las limitaciones de su enfermedad con su actividad diaria. Esta educación entra en juego cuando la enfermedad ya está diagnosticada, su seguimiento a lo largo del tiempo es un requerimiento indispensable y su propósito final se dirige a disminuir las crisis agudas y a evitar las complicaciones (García y Suarez., 2007).
La educación de las personas con Diabetes Mellitus, en la atención primaria de salud, está bien justificada y valorada cuando se cuenta con las competencias que exige el cuidar y enseñar a cuidarse y la comprensión de que solo aquel que aprenda a cuidarse podrá alcanzar una buena calidad de vida. Si el fin último de la educación en diabetes es que la persona pueda controlar su enfermedad y mejorar su calidad de vida, aquella debe conocer la importancia de mantener cifras normales de glicemia, siendo necesaria la comprensión, motivación y destreza práctica para su autocuidado (García y Suarez, 2007 y Pérez et al., 2009).

La intervención educativa por profesionales de enfermería a personas con DM2, para mejorar el autocuidado, fue valorada en México, con la aplicación de la Escala de Capacidades de Autocuidado. Los resultados se analizaron estadísticamente, dentro de un grupo caracterizado en forma predominante por ser de sexo femenino, con baja escolaridad, con cumplimiento de labores en el hogar, casados y dependientes económicamente. Se encontró significancia estadística en la capacidad de autocuidado después de la intervención educativa por enfermería, reflejando así su potencial (Ávila et al., 2006).

De manera similar, en el logro de cambios de comportamiento que definen los estilos de vida de personas con DM2, se revela la importancia de la educación como intervención en el control metabólico de adultos. Esto se acompaña de consejería a las personas con la enfermedad, entendiendo que la adherencia al tratamiento es relativa $y$ depende frecuentemente del "sentimiento de malestar ocasionada por el azúcar". Las herramientas de autocuidado enseñadas para el control de la enfermedad, se señalan decisivas para la regulación de la dieta, la actividad física, la toma de los medicamentos y el aprendizaje del reconocimiento e interpretación de signos y síntomas de alteración individual. Lo anterior mejora la 
toma de decisiones y los resultados de la hemoglobina glucosilada (HbA1c) (Gallegos etal., 2006).

Dentro de los estudios que muestran la importancia del conocimiento sobre la diabetes en las personas con la enfermedad, particularmente uno evalúa, además de los conocimientos, la actitud para afrontar la enfermedad. Dicho estudio señala que no siempre los conocimientos logran cambios de comportamiento, dado que los profesionales de la salud, muchas veces no toman en cuenta lo que la persona con diabetes dice, siente o hace. De esta manera se evidencia la necesidad de reforzar el cuestionamiento sobre las creencias de las personas y las actitudes facilitadoras para el cambio de comportamientos. Para ellos se debe valorar el soporte que reciben los pacientes, por parte de amigos, pares o vecinos; y aumentar la sensibilidad para detectar molestias ocultas y tomar decisiones compartidas, elemento indispensable en el mantenimiento de actitudes positivas para el afrontamiento (Luchetti et al., 2009).

La intervención de enfermería a pacientes diabéticos e hipertensos en un programa de salud familiar, demuestra la importancia del rol educativo en los resultados de salud favorables (Pérez-Cuevas et al., 2009). Dado que existe una valoración positiva de la intervención educativa en salud, con programación y entrenamiento específico de los profesionales de salud, se señalan los beneficios de la actualización en salud de los profesionales. La literatura científica muestra que la educación dirigida a profesionales de enfermería para mejorar el conocimiento del cuidado de las personas con diabetes, logra resultados significativos, valorados estadísticamente, reportando incremento de los conocimientos siempre que fue considerada su participación activa dentro del proceso de aprendizaje (Uriarte et at., 2007). Adicionalmente, se muestra la necesidad de incrementar cursos de educación en diabetes para los profesionales de la salud, considerando las características actuales de la enfermedad, y la caracterización de las personas con DM2. Se trata de personas mayores de 40 años, con mal control metabólico, diagnóstico tardío, con presencia de co-morbilidad, con problemas de viudez, entre otros, que exigen asistencia permanente con comunicación abierta entre profesionales y usuarios de los servicios de salud (Otero et al., 2007; Aguilar-Salinas y Gómez Pérez, 2006).

\section{Percepción de la enfermedad}

La enfermedad genera cambios en las sensaciones y funciones corporales que un individuo puede percibir por sí mismo. La experiencia de la enfermedad es individual y está marcada por los conocimientos individuales y la comprensión de los mismos entorno a la salud-enfermedad. Puede que en una visita de rutina al médico la persona se sienta sana, para descubrir que está oficialmente enfermo, dado los resultados del chequeo (Morrison y Bennett, 2008).

La percepción de aceptación de la enfermedad de personas con DM2 logra mejor afrontamiento y adaptación entendiendo que ésta puede controlarse. En consecuencia, hay mejor calidad de vida. De otra manera, la percepción negativa de la enfermedad produce lesiones en la salud mental de las personas, por lo que a mayor salud mental menor perturbación afectiva demostrada por menor depresión, ansiedad y menores sentimientos de irritabilidad (Vinaccia et al., 2006; Medina et al., 2009; Cid et al., 2006).

Por otra parte, la percepción de la enfermedad desde una óptica familiar, cuando la DM2 forma parte del legado familiar, es valorada a partir de las representaciones que la familia tiene sobre la enfermedad. Cuando ella es diagnosticada en otro familiar, dichas representaciones permiten que se asuma el autocuidado desde la experiencia positiva o 
negativa del familiar que antecedió con la enfermedad (Scollan- Koliopoulos et al., 2006).

La percepción de la enfermedad puede darse también a partir de la comunicación del diagnóstico, momento en el que se deberá aclarar que la enfermedad puede ser controlada con apoyo de los profesionales de salud. Por ello debe existir comunicación permanente en el logro del conocimiento, iniciativa y dedicación de la persona con la enfermedad. Ella puede solicitar ayuda cuando lo considere necesario, situación decisiva en el buen manejo y control de la enfermedad, lo cual impide que se convierta en una situación grave. La persona afectada y la familia deben llevar un proceso de adaptación y afrontamiento ante la nueva situación, y se debe entender la importancia de la atención interdisciplinaria (Díaz y Burguera, 2009; Lazcano-Ortiz et al., 2008).

\section{Síndrome metabólico}

El Síndrome Metabólico (SM) es definido como una asociación de factores de riesgo (intolerancia a la glucosa, obesidad a bdominal, colesterol alto, hipertrigliceridemia e hipertensión arterial) para enfermedad cardiovascular arteriosclerótica y para el desarrollo de DM2. Esta definición es útil para iniciar tratamiento de los factores de riesgo coexistentes, independiente de la presencia de solo uno de ellos. Los factores de riesgo pueden existir ya en la infancia, por lo que es necesario actuar tempranamente en el control de la dieta y en la realización de ejercicio, incrementando recursos en familias, niños e instituciones (Moreno et al., 2009). De otra manera se ha considerado que la asociación de SM y presencia de DM2, no modifica el riesgo cardiovascular, pero se insiste en tratar los factores de riesgo para la prevención de la enfermedad cardiovascular (Church et al., 2009; Olaiz-Fernández et al., 2007).
Adicionalmente, se ha reportado la prevalencia en población hipertensa de SM, situación que exige investigar otros grupos poblacionales para confirmar y extrapolar este resultado, dato de valor en el control de la presencia de factores de riesgo del SM, en personas hipertensas, con el propósito de disminuir la epidemia de enfermedades cardiovasculares y de DM2 (Lombo et al., 2006).

\section{Dieta y ejercicio}

La dieta y el ejercicio controlado son valorados como conductas protectoras de la salud en la persona con DM2, mientras que se valida la relación entre estas conductas protectoras con variables cognoscitivas y afectivas, señalando la importancia de las últimas en el alcance de las primeras (Gallegos y Bañuelos, 2004; Leyva-Moral, 2007).

Así mismo, otros estudios muestran a la intervención con dieta y ejercicio como variables reguladoras de control metabólico, con la aplicación del Modelo de Manejo de Casos para Pacientes con DM2. Eso resulta efectivo en el control glucémico y disminuye el número de pacientes con tratamiento de insulina previa indicación médica, cambiado por tratamiento con hipoglicemiantes orales y complementados con dieta y ejercicio. En este Modelo, la vinculación de la familia a los programas educativos fortalece el apoyo al paciente y por tanto su control glucémico (Martínez et al., 2006; Zanetti et al., 2007)

En forma contraria, se hace evidente la no adherencia al tratamiento no farmacológico, cuando las personas con la enfermedad no cuentan con apoyo sustantivo por el equipo profesional de salud. Se sugiere también educar particularmente a la mujer, quien es la persona que generalmente prepara los alimentos y condiciona los estilos de vida en la alimentación (Medel y González, 2006). 
Las guías de atención a las personas con DM2, de orden nacional e internacional para Latinoamérica, señalan la intervención específica con nutrientes para ser controlados a través de la dieta, que deben ser ajustados por nutricionista de manera individual (Ministerio de Salud de Chile, 2006; República de Colombia, Ministerio de la Protección social, 2006). Se incluye el control del peso acompañado de ejercicio físico, para el mantenimiento del índice de masa corporal ideal (IMC).

Es indispensable que los profesionales de enfermería conozcan y valoren los alimentos utilizados con frecuencia en la región para dar consejería sobre la dieta en el seguimiento de las personas con la enfermedad; conocer los aspectos relacionados con el índice glucémico de los alimentos, la utilización de edulcorantes, los efectos favorables de la fibra, la selección de las grasas, el beneficio de la pérdida de peso, listas de intercambio y sistemas de equivalencia, como fundamento educativo, sin dejar de considerar la importancia de la consulta nutricional individualizada (MacLeallan et al., 2011).

\section{Tratamiento farmacológico - importante adherencia}

La adherencia al tratamiento farmacológico es fundamental para el mantenimiento del control metabólico y la prevención de complicaciones a largo plazo, exige responsabilidades por el equipo de salud y por la persona con la enfermedad. Su seguimiento, control y valoración son importantes en la identificación de alteraciones, para permitir ajustes en cualquier etapa de la enfermedad.

En cuanto al tratamiento farmacológico, las guías de atención a las personas con DM2, de orden nacional e internacional, presentan las recomendaciones que se realizan teniendo en cuenta el nivel de evidencia y sugieren su revisión permanente y actualiza.
La guía de atención a la DM2 de orden nacional, recomienda iniciar el tratamiento farmacológico e ir valorando las metas frente a los resultados del control del paciente. Adicionalmente se propone utilizar las dosis clínicamente útil de los medicamentos, para disminuir reacciones adversas. Entre los antidiabéticos orales, es necesario que se conozcan los secretagogos, aquellos que estimulan la secreción de insulina (sulfonilureas y meglitinidas); los sensibilizantes a la insulina o que disminuyen la insulinoresistencia (biguanidas y tiazolidinedionas); y los inhibidores de alfa glucosidasa intestinal: Acarbosa, que enlentecen la absorción de los hidratos de carbono (MacLeallan et al., 2011).

Por otra parte, la terapia con insulina para DM2 se ha ido renovando a partir de los años noventa. Los protocolos proponen esquemas combinados de dos o tres medicamentos antes de iniciar la terapia con insulina o iniciar en forma más temprana la insulina si la dieta, el ejercicio y la farmacoterapia oral no logran el control glucémico. Tanto la insulina cristalina, como la NPH, se obtenían inicialmente de páncreas de animales. De manera reciente, la ingeniería genética ha conseguido insulina con estructura igual a la humana, insulinas humanas. Se han introducido también insulinas genéticamente modificadas análogas de la insulina humana. Se encuentran unas de acción más rápida que la insulina cristalina (Lispro, Aspart y Glulisina), otras más lentas y estables que la NPH (Glargina y Detemir). Es necesario reconocer las indicaciones y combinaciones para el control de la enfermedad de modo individualizado (MacLeallan et al., 2011. López, 2009).

Así mismo, para el control de la hipertensión arterial (HTA), evento frecuente de comorbilidad en personas con DM2, la intervención farmacológica se hace muchas veces necesaria para mantener cifras por debajo de 130/80 mm de Hg. La presencia de 
HTA multiplica el riesgo de morbilidad, mortalidad y discapacidad en forma exponencial. La terapia inicial para reducir eventos cardiovasculares se realiza con inhibidores de la enzima convertidora de angiotensina (I-ECA), antagonistas de receptores de angiotensina (ARA), $\beta$ diuréticos tiazídicos y bloqueadores de los canales de $\mathrm{Ca}$ (BCC) (MacLeallan et al., 2011; PérezVizcaíno, 2009).

\section{Evaluación y cuidado psicosocial}

El estado psicosocial puede impactar la adherencia al tratamiento, por lo que evaluar su condición se traduce en necesidad fundamental. La educación correspondiente debe ser realizada por personal capacitado, por lo que se insiste en la formación de educadores en DM2. Se deben evaluar las actitudes ante la enfermedad, expectativas ante el tratamiento y resultados, fuentes de financiación; investigar problemas como depresión, desórdenes alimentarios, y de alteración cognitiva, primordialmente cuando la adherencia al tratamiento no es la esperada (MacLeallan et al., 2011).

La diabetes es una enfermedad que requiere comunicación permanente entre las personas con la enfermedad y los profesionales de la salud, debido a la necesidad de los pacientes de tomar decisiones importantes relativas al tratamiento. El conocimiento del paciente y su dedicación al autocuidado es valorado como afrontamiento positivo ante la enfermedad (Lazcano et al., 2008).

Dentro de las causas del manejo inadecuado de la DM2, se expresan circunstancias relacionadas con baja escolaridad de las personas con la enfermedad, las condiciones económicas difíciles y la edad de adulto mayor, entre otras, que interfieren en la correcta interpretación y cumplimiento de las orientaciones y recomendaciones que ofrecen los equipos de salud. El ajuste de las intervenciones al valorar estas circunstancias, tales como las derivadas de las teorías del comportamiento, como son, el modelo de creencias en salud, la autoeficacia, el locus de control, etc. (Pace, 2006).

En Chile, con la consideración del Modelo de Creencias en Salud, en un grupo de personas con DM2, atendidas por consulta externa, se señala que mientras más beneficios se atribuyen al tratamiento, se asegura el control y mientras menos barreras se perciben para asegurar el tratamiento, mayor es la probabilidad de tener un control metabólico adecuado. Las personas del estudio eran adultos menores de 75 años, en su mayoría mujeres y amas de casa, con edad media de 61 años, y con bajos niveles educativos. En el estudio se relaciona control metabólico, compensado o no (de acuerdo al reporte de HbA1c), con variables propias del tratamiento; tiempo del diagnóstico, tipo de tratamiento y estado nutricional, y con variables psicosociales basadas en el Modelo referido (susceptibilidad percibida, severidad percibida, beneficios atribuidos al tratamiento médico y barreras para enfrentar la enfermedad). El estudio valora las contribuciones de los valores subjetivos para diseñar estrategias y tratamientos más integrales (Quintana et al., 2008).

\section{Asistencia hospitalaria de personas con DM2}

Se avala la necesidad de contar con programas estructurados de educación terapéutica en los hospitales, donde son ingresadas las personas con DM2, por causas relacionadas o no con la enfermedad. Muchas veces es requerido el inicio de tratamiento con insulina en forma provisional o definitiva, la educación en este momento, considerando las características específicas de las personas, es fundamental para la prevención de otras complicaciones y el mantenimiento de su salud y calidad de vida (Monteagudo y Segura, 2010). 
Estudios sobre conocimientos de las personas con DM2 hospitalizadas, demuestran que son insuficientes, por lo que se hace necesario incluir dentro de las instituciones hospitalarias, personal de salud que fortalezca los conocimientos para disminuir el ingreso hospitalario y mejorar la calidad de vida de las personas comprometidas con la enfermedad. La aplicación del Modelo de Manejo de Casos en pacientes con DM2 en el ambiente hospitalario, evidencia la disminución de la estancia hospitalaria, los ingresos hospitalarios, la HbA1c; mejora las acciones de autocuidado y la mayor participación familiar (Miyar et al., 2008; Martínez et al., 2006).

\section{Complicaciones de la diabetes mellitus}

Las complicaciones de la DM2 se clasifican en agudas y crónicas, y es necesario prever su desarrollo para poder intervenir de manera apropiada en cada situación específica. La hipoglicemia, la complicación aguda más común y las complicaciones crónicas ocasionadas por afecciones de tipo micro vascular y macro vascular, como retinopatía, nefropatía, neuropatía y enfermedad coronaria, deben ser atendidas de manera oportuna, con el tránsito diligente de requerimientos de apoyo en prevención y tratamiento. El plan de cuidado debe ser interdisciplinar, con apoyo psicológico, nutricional y farmacológico.

Está demostrado que la aparición de complicaciones tiene causalidad múltiple, en donde habría que considerar aspectos genéticos. También se ha hallado labilidad en algunas personas para complicaciones agudas con mayor tendencia a la cetosis o a la hipoglucemia (Mato y Pinal, 2009).

Se hace importante hablar de la prevención del pie diabético, dado que la enfermedad vascular periférica se manifiesta por la alteración circulatoria, complicación que se suma a la de neuropatía. En relación a este planteamiento debe existir valoración permanente de los pies, y educar sobre el cuidado y revisión diaria de los pies (Levy y Calle- Pascual, 2009).

Se menciona la importante valoración de la disfunción eréctil, que se encuentra dentro de las complicaciones crónicas de la DM2, por su impacto en los varones. Existe tratamiento de apoyo por lo que siempre deberá hacerse uso de la consejería médica (Acosta, 2009).

Por último, se menciona la enfermedad periodontal (EP), como complicación significativa de la Diabetes Mellitus. Un mal control de la glicemia es factor de riesgo para la EP. De manera inversa, también existe evidencia de que la EP puede afectar el buen control de la glicemia. Se señala el bajo conocimiento de las personas con DM2, sobre la importancia del cuidado bucodental que puede influir en la poca prevención de factores de riesgo para la enfermedad, así como la no adecuada interpretación de las orientaciones y prácticas de higiene, posiblemente debida a dificultades de lectura, escritura y comprensión (Juárez et al., 2007; Kamran etal., 2007).

Finalmente, considerar la complejidad de la enfermedad y conocer la complejidad de la atención interdisciplinaria, abrirá oportunidades para mejorar la calidad de vida de las personas comprometidas con la enfermedad. 


\section{Referencias}

Acosta L. Disfunción eréctil y diabetes Mellitus. 2009. En: La diabetes Mellitus en la práctica clínica. Tebar FJ, Escobar F. Capítulo 33. Panamericana: Buenos Aires, Argentina. pp.262 -267.

Aguilar-Salinas CA, Gómez-Pérez FJ. Declaración de Acapulco: Propuesta para la reducción de la incidencia de la Diabetes en México. 2006. Revista de investigación clínica. [Consultado Noviembre 15 de 2011]. Vol 58(1):71-77.Disponible en: http://www.scielo.org.mx/pdf/ric/v58n1/v5 8n1a9.pdf

Aponte-Garzón LH, Hernández-Páez RF. Conocimiento de los profesionales de enfermería sobre Diabetes Mellitus Tipo 2, Villavicencio, Meta, 2010. Revista Orinoquia. 2010;16(1):93 -109.

Ávila H, Meza S, Frías B, Sánchez E, Vega C, Hernández MA. 2006. Intervención de enfermería en el autocuidado con apoyo educativo en personas con Diabetes Mellitus tipo 2. Cultura de los cuidados. \{Consultado 29 de octubre de 2011\} Vol. 10(20) [Aprox. 16 pantallas]. Disponible en:http://rua.ua.es/dspace/bitstream/100 45/877/1/culturacuidados_20_20.pdf

Cid P, Merino JM, Stiepovich J. 2006. Factores biológicos y psicosociales predictores del estilo de vida promotor de salud. Rev. méd. Chile [revista en la Internet]. [citado 2011 Jul 27]; 134(12): [14 pantallas] Disponible en: URL: http:// www.scielo.cl/scielo. php? script $=$ sci_arttext\&pid $=$ S00 34 98872006001200001\&lng=es.doi:10.406 7/S0034-988720 06001200001.

Commers M, Gottlieb M, Kock G. 2006. How to change enviromental conditions for health. Health Promotion International [consultado noviembre 3 de 2011] Vol 22(1): 80 -87. Disponible en: http://heapro .oxfordjournals.org/content/22/1/80.full.pd $\mathrm{f}+\mathrm{html}$ ? sid = 3a6ca3f3-31c9-4088-87862a87f56bed6b

Church TS, Thompson A, Katzmarzyk P, Sui X, Neil J, Conrad E et al. 2009. Metabolic Syndrome and Diabetes, alone and in combination, as predictors of cardiovascular disease. Diabetes Care, 32, (7); ProQuest Nursing \& Allied Health Source. [Aprox. 20 pantallas] [consultado 17 de noviembre de 2011]. Disponible en: http://search.proquest.com/nursing/docvie w/223024039/fulltextPDF/133177F1B9F66 269C $2 / 2$ ?accountid $=48773$

Díaz S, Burguera B. 2009. Actitud ante el debut de una diabetes Mellitus. Perspectiva general del tratamiento. En: La diabetes Mellitus en la práctica clínica. Tebar FJ, Escobar F. Capítulo 8. Panamericana: Buenos Aires, Argentina. pp.77 -84.

Gallegos E, Bañuelos Y. 2004. Conductas protectoras de salud en salud en adultos con Diabetes Mellitus tipo II. Investigación y Educación en Enfermería - Medellín, Vol. 22 n. 2. Universidad de Antioquia. Medellín, Colombia. [Aprox. 23 pantallas] [Consultado noviembre 17 de 2011]. Disponible en: http://revinut.udea.edu.co /index.php/iee/article/view/2955/2648

Gallegos E, Ovalle-Berúmen F, Gómez-Meza MV. 2006. Metabolic control of adults with tipe 2 Diabetes Mellitus through education and counseling. Clinical Scholarship. Journal of Nursing Scholarship, 38 (4); ProQuest Nursing \& Allied Health Source [aprox. 18 pantallas] [Consultado noviembre 15 de 2011] Disponible en: http://search.proquest.com/nursing/docvie w/236403562/fulltext/1330D0E8EBD1D2F 9339/1 ? accountid $=48773$ 
García R, Suárez R. 2007.La educación terapéutica. Propuesta de un modelo teórico desde la experiencia del programa cubano de educación en Diabetes. OPS/OMS. (Documento Oficial No. 325) $17^{\mathrm{a}}$. Ed. Washington D.C. pp. 102. Disponible en: http://www. paho.org/ spanish/ad/dpc/nc/dia-cuba.pdf

García R, Suárez R. 2007. La educación a personas con diabetes mellitus en la atención primaria de salud. Rev Cubana Endocrinol [revista en la Internet]. [citado 2011 Nov 07]; 18(1). Disponible en: http://scielo.sld.cu /scielo.php?script=sci_ arttext\&pid = S1561-29532007000100 $005 \& \operatorname{lng}=$ es.

Gobierno de Chile. Ministerio de salud. 2006. Guía Clínica. Diabetes Mellitus Tipo 2. Gobierno de Chile. Serie guías clínicas minsal $n^{\circ} x x, 1^{a}$ edición. Santiago: Minsal. Disponible en: http://www.redsalud. gov.cl/archivos/guiasges/diabetesGes.pdf

Higashida B. 2008. Educación para la salud. Tercera Ed. Mc Graw Hill. pp.239.

Juárez R, Chahin JR, Vizcaya M, Arduña El. 2007. Conocimientos, comportamientos, percepciones y actitudes concernientes a la salud oral entre pacientes diabéticos. Rev Cubana Estomatol [revista en la Internet]. [citado 2011 Nov 15]; 44(2). Disponible en: http://scielo.sld.cu/scielo.php? script=sci_arttext\&pid=S0034-75072007 $000200004 \& \operatorname{lng}=\mathrm{es}$.

Kamran M, Ayyaz Ak, Munawar MA, Saima C. 2007. Oral Health Knowledge, attitude and pratices and sources of information for diabetic patients in Lahore, Pakistan. Epidemiology Services Research Diabetes Care, 30 (12); ProQuest Nursing \& Allied Health Source. [Aprox. 8 pantallas] [consultado 17 de noviembre de 2011].
Disponible en: URL: http://search. proquest.com/nursing/docview/223044715 /fulltextPDF/1331768B04E4898E86D/1 ?ac countid $=48773$

Lazcano M, Salazar BC, Gómez MV. 2008. Validación del instrumento Afrontamiento y proceso de adaptación de Roy en pacientes con Diabetes Mellitus tipo 2. Aquichan [en línea] [Consultado 2011 Julio 31] 8 (1):116 - 125. Disponible en: URL: http://pensa mientoycultura.unisabana.edu.co/sabana/i ndex.php/aquichan/article/view/128/256

López M. 2009. La insulina en el tratamiento de la Diabetes Mellitus. En: La diabetes Mellitus en la práctica clínica. Tebar FJ, Escobar F. Capítulo 13. Panamericana: Buenos Aires, Argentina. pp.103-113.

Levy A, Calle-Pascual A. 2009. Los problemas en los pies en las personas con diabetes. En: La diabetes Mellitus en la práctica clínica. Tebar FJ, Escobar F. Capítulo 44. Panamericana: Buenos Aires, Argentina. pp.367 -374.

Leyva-Moral JM. 2007. La entrevista motivacional como instrumento para promover la actividad física y la adhesión dietética en personas con diabetes: revisión bibliográfica. Nure Investigación, $\mathrm{n}^{\circ} 29$. En Línea. [Consultado 19 de noviembre de 2011]. Disponible en: http://www. nureinvestigacion.net/FICHEROS_ADMINI STRADOR/ORIGINAL/pdf_original_29.pdf

Lombo B, Villalobos C, Tique C, Satizábal C, Franco C. 2006. Prevalencia del síndrome metabólico entre los pacientes que asisten al servicio Clínica de Hipertensión de la Fundación Santa Fe de Bogotá. Revista Colombiana de Cardiología. [consultado noviembre 17 de 2011] Vol 12(7): 472 -478. Disponible en: http://www.scc.org.co /Portals/0/v12n7a4.pdf 
López M. 2009. La insulina en el tratamiento de la Diabetes Mellitus. En: La diabetes Mellitus en la práctica clínica. Tebar FJ, Escobar F. Capítulo 13. Panamericana: Buenos Aires, Argentina. pp.103-113.

Luchetti FF, Zanetti ML, Santos MA, Martins TA, Sousa D, Teixeira CR. 2009. Conocimiento y actitudes: componentes para la educación en diabetes. Rev. LatinoAm. Enfermagem [serial on the Internet]. [cited 2011 Nov 16]; 17(4): 468-473. Available from: http://www.scielo. br/scielo.php?script $=$ sci_arttext $\&$ pid $=$ S0 10 4-11692009000400006\&Ing =en . http://dx.doi.org/10.1590/S010411692009000400006.

MacLeallan D, Morley C, Traviss K, Cividin T. 2011. Toward evidence- based, clientcentred nutrition education guidelines: Dietitian and consumer survey results. Canadian Journal of Dietetic Practice and Research, 72(3), 111-116. Retrieved from: http://search.proquest.com/docview/90438 5832 ? accountid $=48773$

Martínez MG, Moreno MG, Sauceda PF, Vásquez L, Medina SG, Zamarripa AL. 2006. Modelo de manejo de casos en pacientes con Diabetes Mellitus Tipo II. Invest. educ. enferm vol.24 no.1 Medellín. Disponible en: URL: http://www.sci. unal.edu.co/scielo.php?script $=$ sci_arttext \& pid = S0120-53072006000100006\&Ing $=$ en $\& n r m=i s o \& t \operatorname{lng}=e s$

Mato J, Pinal I. 2009. Complicaciones de la diabetes Mellitus. Clasificación y enfoque general. En: La diabetes Mellitus en la práctica clínica. Tebar FJ, Escobar F. Capítulo 24. Panamericana: Buenos Aires, Argentina. pp.189-195.

Medina OM, Díaz AD, Barrientos E, Peña D. Percepción de la salud y su efecto en pacientes con diabetes. av. Enferm. 2009.
[Consultado 17 de julio 2011]; 27 (2): [17 pantallas] Disponible en: URL: http://www.enfermeria.unal.edu.co/revista /articulos/xxvii2_2.pdf

Medel BC, González L. 2006. Estilos de vida: Experiencias con la adherencia al tratamiento no farmacológico en diabetes mellitus. Index Enferm [revista en la Internet]. [citado 2011 Nov 18]; 15(5253): 16-19. Disponible en: http://scielo .isciii.es/scielo.php?script=sci_arttext\&pid $=$ S1132-12962006000 100004\&Ing=es. http://dx.doi.org /10.4321/S1132-1296200 6000100004

Mendoza Ma, Welbeck M, Parikh G. 2010. Behavioral and educational Approaches to diabetes self- management. In: Principles of diabetes Mellitus. Chapter 40. Editor: Poretsky Leonid. Second edition. New York, USA. pp. 659-675

Milla N, Perez JR, Malaga JE, et al. 2008. Conocimientos sobre su enfermedad en pacientes con diabetes mellitus tipo 2 que acuden a hospitales generales. Rev Med Hered. [online]. Vol.19, no.2 [citado 20 Noviembre 2011], p.46-47. Disponible en: http://www.scielo.org.pe/scielo.php?script =sci_arttext $\&$ pid $=$ S1018-130X200 000200 $005 \&$ Ing $=e s \& n r m=i s o>$. ISSN 1018-130X.

Ministerio de la protección social. OPS. Indicadores básicos de salud 2009. Bogotá, Colombia.

Miyar Otero L, Zanetti ML, Daguana Ogrizio M. 2008. Knowlegde of diabetic patiens about their disease before and after implementing a diabetes education program. Rev Latino- am enfermagen, 16(2): 231 - 7. [Consultado noviembre 19 de 011]. Disponible en: http://www. docstoc.com/docs/69257959/KNOWLEDG E-OF-DIABETIC-PATIENTS-ABOUTTHEIR-DISEASE-BEFORE-AND 
Monteagudo AM, Segura JM. 2010. Implementación de un programa de educación terapéutica mínimo para pacientes diabéticos hospitalizados que inician tratamiento con insulina. Av Diabetol, 26: 281 -285 [Consultado noviembre 19 de 2011]. Disponible en: http://www.elsevier.es/sites/default/files/els evier/pdf/326/326v26n04a90000522pdf00 1 .pdf

Moreno B, Zugasti A, Jimenez A. 2009. Síndrome Metabólico. Concepto, patogenia y diagnóstico. Actitud Clínica. En: La diabetes Mellitus en la práctica clínica. Tebar FJ, Escobar F. Capítulo 8. Panamericana: Buenos Aires, Argentina. pp. 59-67.

Morrison V, Bennett P. Psicología de la salud. Pearson Prentice Hall. 2008. Madrid, España. pp. 728

OMS. Organización Mundial de la Salud Ministerio de la Protección Social - Instituto Nacional de Salud. 2010. Indicadores Básicos 2010. Situación de Salud en Colombia. Bogotá, Colombia. p. 8

Olaiz-Fernández G, Rojas R, Aguilar-Salinas CA, Rauda J, Villalpando S. 2007. Diabetes mellitus en adultos mexicanos: resultados de la Encuesta Nacional de Salud 2000. Salud pública Méx [serial on the Internet]. [cited 2011 Nov 18]. Available from: http://www.scielosp.org/scielo.php?script= sci_arttext\&pid = S0036-3634200700090 0004\&lng=en.http://dx.doi.org /10.1590/ S0036-36342007000900004.

Otero LM, Zanetti ML, Teixeira CR. 2007. Características sociodemográficas y clínicas de una población diabética en el nivel primario de atención a la salud. Rev. LatinoAm. Enfermagem [serial on the Internet]. [cited 2011 Nov 16]; 15(spe): 768-773. Available from: http://www.scielo.br /scielo. php? script=sci_arttext\&pid $=$ S010411692007000700 009\&Ing=en.http:// dx.doi.org /10.1590/S0104-11692007000 700009 .

Pace AE, Ochoa-Vigo K, Caliri MH, Fernandes AP. 2006. El conocimiento sobre diabetes mellitus en el proceso de autocuidado. Rev. Latino-Am. Enfermagem [serial on the Internet]. [cited 2011 Nov 18]; 14(5): 728-734. Available from: http://www. scielo.br/scielo.php?script=sci_arttext\&pid $=$ S0104-1169200600050 0014\&Ing=en. http://dx.doi.org/ 10.1590/S010411692006000500014.

Pérez A, Alonso L. García AJ, Garrote I, González S, Morales JM. 2009. Intervención educativa en diabéticos Tipo II. Revista Cubana de Medicina General Integral, 25(4)17-29 [Consultado noviembre 15 de 2011]. Disponible en: http://bvs.sld.cu/revistas/mgi/vol_25_4_09 /mgi03409.pdf

Pérez-Cuevas R, Reyes $\mathrm{H}$, Vladislavovna $\mathrm{S}$, Zepeda M, Díaz G, Peña A, Muñoz O. 2009. Atención integral de pacientes diabéticos e hipertensos con participación de enfermeras en medicina familiar. Rev Panam Salud Pública, 26(6):511-7. \{Consultado 29 de octubre de 2011\} Disponible en: http://www.scielosp.org/ pdf/rpsp/v26n6/06.pdf

Pérez-Vizcaíno F, Cogolludo A, Tamargo J. 2009 . Fármacos vasodilatadores. Antagonistas de los canales de calcio. Fármacos antihipertensivos. Capítulo 25. En: Velazquez L, Moreno A, Lizasoain I, Leza JC, Moro MA, Portolés A. Velázquez Farmacología Básica y Clínica. Panamericana. 18 a . Ed. Buenos Aires, Argentina. pp. 419 -439.

Quintana AA, Merino JM, Merino RP, Cea JC. 2008. Variables psicosociales asociadas a

Educación en diabetes: un aspecto clave de la formación actual en enfermería 
compensación metabólica de pacientes diabéticos de tipo 2. Rev. méd. Chile [revista en la Internet]. [citado 2011 Nov 18]; 136(8): 1007-1014. Disponible en: http://www.scielo.cl/scielo. php?script=sci _arttext\&pid=S0034-98872008000800007 \&lng =es.doi:10.4067/S0034-98872008 000800007.

República de Colombia. Ministerio de la Protección Social. 2006. Guía 17. Guía de atención de la Diabetes Mellitus Tipo 2. Guías y normas 412 - Tomo 2 - Parte 2. Pp. 371 -439. Disponible en: http://www. nacer.udea.edu.co/pdf/libros/guiamps/guia s17.pdf

Scollan-Koliopoulos M, O'Connell K, Wlaker EA. 2005/2006. Assessing legacies of Diabetes: Recolletions of family members' illness representations and outcomes Journal of Theory Construction \& Testing, 9, 2; ProQuest Career and Technical Education: Health \& Medicine [ consultado noviembre 16 de 2011] [Aprox. 24 pantallas] Disponible en: http://search. proquest.com/careerhealth/docview/21921 5064/13312BB37ED2D525D14/10?accou ntid $=48773$

Severo T, Oramas R, González L. 2007. Evaluación del efecto de una intervención educativa en los pacientes diabéticos de cuatro consultorios. Rev Cubana Med Gen Integr [revista en la Internet]. [citado 2011 Nov07];23(2):Disponible en: http:// scielo.sld.cu/scielo.php?script=sci_arttext $\& p i d=S 0864-21252007000$ $200007 \& \operatorname{lng}=$ es.

Uriarte S, Flores P, Castro AN. 2007. Conocimiento de enfermería en el cuidado de pacientes diabéticos antes y después de una intervención educativa. Enfermería global. No. 11. Revista electrónica semestral de enfermería.[Aprox.18 pantallas].Disponible en: http://dialnet. unirioja.es/servlet/articulo? codigo $=238$ 5625
UNILLANOS - Universidad de los Llanos. Programa de Enfermería. 2011. Documento Renovación Registro Calificado. Facultad Ciencias de la Salud. Villavicencio, Colombia. pp. 189.

Vinaccia S, Fernández $\mathrm{H}$, Escobar $\mathrm{O}$, Calle E, Andrade I, Contreras F, Tobón S. 2006. Calidad de vida y conducta de enfermedad en pacientes con diabetes Mellitus tipo II. Suma Psicológica, Vol. $13 \mathrm{~N}^{\circ} 1$. [aprox. 46 pantallas] [Consultado Noviembre 15 de 2011] Disponible en: http://pepsic. bvsalud.org/pdf/suma/v13n1/v13n1a03.pdf

WHO - World Health Organization. 2011. About the diabetes programme. [Página de internet]. Ginebra. [Consultado 28 de Octubre 2011]. Disponible en: http://www.who.int/diabetes/en/

Zanetti Maria Lúcia, Otero Liudmila Miyar, Biaggi Marília Voltolini, Santos Manoel Antônio dos, Péres Denise Siqueira, Guimarães Fernanda Pontin de Mattos. 2007. Satisfaction of diabetes patients under follow-up in a diabetes education program. Rev. Latino-Am. Enfermagem [serial on the Internet]. [cited 2011 Nov 07]; 15(4): 583-589. Available from: URL: http://www.scielo.br/scielo.php?script=sci _arttext\&pid=S0104-116920070 $00400010 \&$ Ing = en.http://dx.doi. org/10.1590/S0104-11692007000400010 